\title{
PARÂMETROS DENDROMÉTRICOS E CORRELAÇÕES COM PROPRIEDADES TECNOLÓGICAS EM UM HÍBRIDO CLONAL DE Eucalyptus urophylla $x$ Eucalyptus grandis ${ }^{1}$
}

\author{
Fabricio Gomes Gonçalves², José Tarcísio da Silva Oliveira², Gilson Fernandes da Silva², Mauro Eloi \\ $\mathrm{Nappo}^{3}$ e Mario Tomazelo Filho ${ }^{4}$
}

\begin{abstract}
RESUMO - A qualidade da madeira aparentemente está associada às características dendrométricas, que por sua vez são influenciadas pelas condições de crescimento. Os objetivos deste estudo foram a caracterização dendrométrica e a realização de correlações de Pearson entre alguns parâmetros tecnológicos da madeira do híbrido clonal de Eucalyptus urophylla x Eucalyptus grandis sob três estratos silviculturais. Os dados dendrométricos foram analisados mediante a análise de regressão. Os resultados mostraram que o híbrido apresenta comportamento similar ao das demais madeiras da mesma espécie que cresceram em outras regiões, destacando-se a espessura do alburno no E1 (talhadia simples, 70 meses e com um desbaste), com pouca variação ao longo do fuste. Todas as árvores apresentaram, em geral, baixa conicidade ao longo do fuste e baixo volume de casca entre os estratos. Na correlação de Pearson foram avaliados entre si nove parâmetros dendrométricos, sete físicos e três mecânicos, longitudinalmente em seções de 3 m e na posição medula-casca nas duas primeiras toras. Os coeficientes encontrados demonstram haver fortes correlações entre as propriedades, destacando-se a densidade básica em relação às demais características e o coeficiente de anisotropia, ambas importantes no processo de desdobro e secagem da madeira. Não houve correlações significativas nas contrações lineares e volumétricas ao longo do comprimento do fuste. A variação radial mostrou boas correlações entre todas as propriedades mecânicas avaliadas nas duas primeiras toras. Em contrapartida, as propriedades físicas avaliadas na posição radial não apresentaram correlações significativas.
\end{abstract}

Palavras-chave: Eucalyptus urophylla x Eucalyptus grandis, Correlações propriedades da madeira e qualidade da madeira.

\section{DENDROMETRIC PARAMETERS AND CORRELATIONS WITH TECHNOLOGICAL PROPERTIES IN A CLONAL HYBRID OF Eucalyptus urophylla $x$ Eucalyptus grandis}

\begin{abstract}
Wood quality is apparently associated with the dendrometric characteristics that are influenced by growth conditions. The aim of this study was the dendrometric characterization and realization of Pearson Correlations among some technological parameters in the clonal hybrid wood Eucalyptus urophylla $x$ Eucalyptus grandis grown in three silvicultural extracts. The dendrometric data were analyzed by regression analysis. The results show that the hybrid presents similar behavior to other eucalypt of the same species grown in other places with sapwood thickness in E1 (coppice with 70 months and one thinning), with little variation along the stem. All evaluated trees presented, in general, a low taper along the stem with very little bark volume among the extracts. The Pearson Correlation studied nine dendrometric parameters, seven physical and three mechanical properties. The parameters were evaluated, longitudinally, in three meter sections along the stem and in the pith-bark position in the first two logs according to the Pearson's Correlation. The coefficients demonstrated a strong correlation among the properties, with basic density being highly correlated to the other characteristics and anisotropic coefficient, important in the process of sawing and drying wood, as well as sapwood thickness. The correlation to the height relationship wasn't significant to the linear and volumetric shrinkage. Radial variation showed a good correlation among all the evaluated mechanical properties in the two first logs. On the other hand, the physical properties in the radial position didn't present significant correlations with tangential shrinkage.
\end{abstract}

Keywords: Eucalyptus urophylla x Eucalyptus grandis, Correlation of wood properties and wood quality.

\footnotetext{
${ }^{1}$ Recebido em 08.05.2008 e aceito para publicação em 02.03.2010.

${ }^{2}$ Universidade Federal do Espírito Santo, UFES, Brasil. E-mail: <fabriciogomes@ibest.com.br>, <jtsilva@ndp.ufes.br>, <gfsilva2000@yahoo.com.br>.

${ }^{3}$ Universidade de Brasília, UNB, Brasil. E-mail: <mauronappo@yahoo.com.br>.

${ }^{4}$ Universidade de São Paulo, Escola Superior de Agricultura Luiz de Queiroz, Brasil. E-mail: <mtomazel@esalq.usp.br>.
} 


\section{INTRODUÇÃO}

O comportamento de uma floresta plantada, no que se refere aos tipos diferenciados de manejo, pode proporcionar alterações na qualidade da madeira, influenciando no produto final desejado e, consequentemente, no seu valor comercial. A busca por material de qualidade superior que atenda ao exigente mercado madeireiro vem crescendo a cada ano. Tal comportamento é refletido nos diferentes segmentos florestais a partir da silvicultura e do melhoramento florestal, finalizando no setor tecnológico de base florestal. Assim, o conhecimento da variabilidade entre os materiais disponíveis e as relações entre as características tecnológicas e dendrométricas são de extrema importância.

Em florestas plantadas, diversas são as atividades silviculturais que devem ser observadas quando o objetivo é a produção de madeira sólida de qualidade. As práticas mais importantes são: a densidade de plantio, o desbaste e a desrama, as quais podem definir a qualidade da madeira. Têm importância o preparo do solo e a adubação, que também podem modificar o comportamento celular da árvore e, consequentemente, a sua qualidade (SCHÖNAU e COETZEE, 1989; WILKINS e HORNE, 1991; ZOBEL, 1992; PINHEIRO, 1999; FINGER et al., 2001; BERGER et al., 2002; GONÇALVES et al., 2004; LEITE et al., 2004).

A idade das árvores também contribui para modificar as características da madeira, influenciando na quantificação do volume, na forma da árvore, no diâmetro à altura do peito e nas relações cerne-alburno. A conicidade de uma árvore pode ser afetada pela espécie (exemplares de florestas plantadas com essências exóticas tendem a ser mais cônicos que de espécies nativas) ou pela idade (árvores mais velhas apresentam menor conicidade) (SCOLFORO e FIGUEIREDO FILHO, 1993) ou, ainda, de acordo com Grosser (1980 citado por SCANAVACA Jr. e GARCIA, 2003), pela densidade de plantio (em espaçamentos maiores, há tendência em formar árvores com conicidade maior).

De acordo com Ribeiro Júnior (2001), a determinação de correlações entre duas diferentes variáveis permite o conhecimento de quanto essas variáveis estão próximas. Adorno e Garcia (2003), estudando correlações lineares entre as principais características tecnológicas da madeira de clones de Eucalyptus grandis e E. urophylla, ambas com 8 anos de idade, afirmaram que a densidade básica apresentou correlação negativa com as variáveis do processamento mecânico da madeira de E. urophylla.
Assim, pode-se dizer que há meios de, através da densidade, conhecer e propor soluções em programas de melhoramento genético que visam à redução das flechas observadas durante o desdobro das árvores.

Este trabalho teve como objetivo estudar algumas características dendrométricas e suas relações de interdependência entre as características dendrométricas e as propriedades físicas e mecânicas do híbrido clonal de Eucalyptus urophylla x Eucalyptus grandis em duas diferentes idades, provenientes de talhadia simples e reforma.

\section{MATERIAL E MÉTODOS}

Os dados e material para este estudo foram coletados em um experimento com clones híbridos de Eucalyptus urophylla x Eucalyptus grandis, de propriedade da empresa Aracruz Celulose S.A., localizado no Sul do Estado da Bahia, Municípios de Mucuri e Nova Viçosa. O solo local é pertencente à Formação Barreiras, sendo predominante nos três estratos estudados o Podzólico Amarelo Distrófico. De acordo com a classificação climática de Köppen, há predomínio do tipo “Af”, sendo o clima tropical chuvoso e quente, com temperatura superior a $18^{\circ} \mathrm{C}$ no mês mais frio. A precipitação anual está em torno de 1.378,0 mm, e no mês mais seco ocorre precipitação em torno de 60,0 mm. A região Sul do Estado da Bahia apresenta déficit hídrico em torno de $124,0 \mathrm{~mm}$ e excedente próximo a 78,0 mm anuais (com base em série histórica de apenas 10 anos).

Foram selecionadas 15 árvores com diâmetro à altura do peito (DAP), entre 28,0 e 30,0 cm, e mensuradas a altura total até um diâmetro de 15,0 cm, além da realização de uma cubagem rigorosa pelo método de Smalian. A Tabela 1 apresenta de forma resumida as características gerais dos estratos avaliados neste estudo.

Os discos de aproximadamente 4,0 cm de espessura, coletados em intervalos de 3,0 m até um diâmetro mínimo de 15,0 cm, foram armazenados em sacos plásticos hermeticamente fechados e transportados para o Laboratório de Ciência da Madeira - LCM, do Núcleo de Estudos e de Difusão de Tecnologia em Floresta, Recursos Hídricos e Agricultura Sustentável - NEDTEC, do Centro de Ciências Agrárias da Universidade Federal do Espírito Santo (CCA/UFES), localizado no Município de Jerônimo Monteiro, ES. 
Tabela 1 - Características gerais dos estratos estudados. Table 1 - General characteristics in the studied extracts.

\begin{tabular}{lccr}
\hline CARACTERÍSTICAS & & ESTRATOS & E3 \\
\cline { 2 - 4 } & E1 & $03 / 1991$ & $06 / 1999$ \\
\hline Época de plantio & $03 / 1999$ & Reforma & Reforma \\
Condução de plantio & Talhadia & $3,0 \times 3,0$ & $6,0 \times 2,5$ \\
Espaçamento (m) & $3,0 \times 3,0$ & 07 & 01 \\
Capina química (unidade) & 04 & 04 & 04 \\
Roçada manual (unidade) & 04 & 166 & 70 \\
Idade de corte (meses) & 70 & 117 e 153 & 42 \\
Desbaste (meses) & 30 & 01 & 01 \\
N $^{\circ}$ de cepas (a partir de 9 meses) & 01 & 364,07 & 119,27 \\
Volume de madeira/ha (m³) & 169,01 & 325 & 150 \\
Densidade na época do corte (árv/ha) & 150 & & \\
\hline
\end{tabular}

Os ensaios físicos e mecânicos foram realizados no LCM/NEDTEC/CCA/UFES, em uma máquina universal de ensaios com aquisição automática de dados, sendo para isso utilizadas as duas primeiras toras por árvore com 3 m de comprimento cada uma, obedecendo ao determinado pela Norma MB-26/40 (ABNT, 1940).
As propriedades físicas foram determinadas de acordo com VITAL (1984), no caso da densidade básica média. As contrações radial, tangencial e volumétrica, bem como o coeficiente de anisotropia, foram determinadas nas duas primeiras toras. A Tabela 2 apresenta os caracteres avaliados, bem como o número de medições de cada estrato.

Tabela 2 - Propriedades físicas, mecânicas e características dendrométricas estudadas na determinação do Coeficiente de Pearson. Table 2-Physical and mechanical properties and dendrometric characteristics studied in the Pearson determination of Coefficient.

\begin{tabular}{|c|c|c|c|c|c|}
\hline \multirow[t]{2}{*}{ CARACTERÍSTICA } & \multirow[t]{2}{*}{ ABREVIAÇÃO } & \multirow[t]{2}{*}{ UNIDADE } & \multicolumn{3}{|c|}{ OBSERVAÇÕES } \\
\hline & & & $\mathrm{E}^{1}{ }^{1}$ & E2 & E3 \\
\hline \multicolumn{6}{|c|}{ PROPRIEDADES FÍSICAS } \\
\hline Densidade básica & DB & $\mathrm{g} / \mathrm{cm}^{3}$ & 235 & 278 & 221 \\
\hline $\begin{array}{l}\text { Densidade aparente no teor de equilíbrio } \\
\text { higroscópico }(16,73 \%)\end{array}$ & DA & $\mathrm{g} / \mathrm{cm}^{3}$ & 235 & 278 & 221 \\
\hline Contração radial & CA & $\%$ & 231 & 257 & 220 \\
\hline Contração tangencial & $\mathrm{CT}$ & $\%$ & 231 & 257 & 220 \\
\hline Contração volumétrica & $\mathrm{CV}$ & $\%$ & 231 & 257 & 220 \\
\hline Fator anisotrópico & FA & - & 231 & 257 & 220 \\
\hline Deformação Residual Longitudinal - DRL & DRL & $\mathrm{mm}$ & 20 & 20 & 20 \\
\hline \multicolumn{6}{|c|}{ PROPRIEDADES MECÂNICAS } \\
\hline Módulo de elasticidade & MOE & $\mathrm{Kgf} / \mathrm{cm}^{2}$ & 134 & 129 & 116 \\
\hline Módulo de ruptura & MOR & $\mathrm{Kgf} / \mathrm{cm}^{2}$ & 134 & 129 & 116 \\
\hline Compressão paralela às fibras & $\mathrm{CP}$ & $\mathrm{Kgf} / \mathrm{cm}^{2}$ & 134 & 129 & 116 \\
\hline \multicolumn{6}{|c|}{ PARÂMETROS DENDROMÉTRICOS E FENOTÍPICOS } \\
\hline Índice de circularidade & IC & - & 40 & 45 & 36 \\
\hline Espessura de alburno & EA & $\mathrm{cm}$ & 35 & 40 & 31 \\
\hline Conicidade & $\mathrm{CO}$ & $\mathrm{cm} / \mathrm{m}$ & 30 & 35 & 30 \\
\hline Fator de forma & FF & - & 0,50 & 0,52 & 0,51 \\
\hline Relação cerne-alburno & RCA & - & 35 & 40 & 31 \\
\hline Porcentagem de casca & PCAS & $\%$ & 35 & 40 & 31 \\
\hline Altura total & $\mathrm{HT}$ & $\mathrm{m}$ & 32,31 & 37,73 & 30,50 \\
\hline Altura comercial & $\mathrm{HC}$ & $\mathrm{m}$ & 20,58 & 24,14 & 18,93 \\
\hline Altura do primeiro galho verde & HPGV & $\mathrm{m}$ & 12,24 & 9,64 & 11,26 \\
\hline
\end{tabular}

E1 - Talhadia (70 meses); E2 - Reforma (166 meses); e E3 - Reforma (70 meses).

E1 - Coppice (70 months), E2 - Reform (166 months), and E3 - Reform (70 months). 
O coeficiente de Pearson foi obtido através do software SAEG, mediante a análise de correlação entre algumas propriedades, listadas na Tabela 2. Apenas os dados médios de cada árvore ou de cada tora foram utilizados na análise de correlação, independentemente do estrato. A determinação do coeficiente de Pearson é importante quando se pretende avaliar a relação entre algumas variáveis para a utilização da madeira como produto sólido em serraria.

Os dados foram analisados mediante a estatística descritiva e a análise de regressão, tendo em vista o fato de o material disponibilizado para o estudo apresentar efeitos que não puderam ser controlados como material genético, sítio (variação física e química), idades diferentes, métodos de implantação (talhadia e reforma do povoamento) e manejo diferenciado. Foram empregados os seguintes modelos para ajustamento de equações aos dados observados:

$$
\begin{aligned}
& Y=\beta_{0}+\beta_{1} X_{i}+\varepsilon \\
& Y=\beta_{0}+\beta_{1} X_{i}+\beta_{2} X_{i}^{2}+\varepsilon \\
& Y=\beta_{0}+\beta_{1} X_{i}+\beta_{2} X_{i}^{2}+\beta_{3} X_{i}^{3}+\varepsilon \\
& Y=\beta_{0}+\beta_{1} \sqrt{X_{i}}+\beta_{2} X_{i}+\varepsilon \\
& Y=\beta_{0} \times X_{i}^{\beta_{1}}+\varepsilon \\
& Y=\beta_{0} \times \beta_{1}^{X_{i}}+\varepsilon \\
& Y=\beta_{0}+\beta_{1} \sqrt{X_{i}}+\beta_{2}+\beta_{3} X^{1,5}+\varepsilon
\end{aligned}
$$

em que $Y_{i}=$ valor médio da propriedade avaliada (variável dependente); $X_{i}=$ valor a ser definido com base na propriedade a ser estimada (variável independente); $\beta_{0} \beta_{1} \beta_{2}$ e $\beta_{3}=$ parâmetros de regressão; e $\varepsilon=$ erro aleatório.

Para a seleção dos modelos, avaliaram-se o coeficiente de determinação ajustado $\left(r^{-2}\right)$ e a significância dos coeficientes de regressão por meio do teste " $t$ ", de Student, em nível de significância de 5\% de probabilidade.

\section{RESULTADOS E DISCUSSÃO}

Os valores encontrados do volume de casca, com base na cubagem rigorosa realizada pelo método de Smalian, são apresentados na Tabela 3.
Salienta-se que, apesar de as árvores apresentarem volumes de casca com valores pouco diferenciados, as médias dos estratos mantiveram-se muito próximas. Destaca-se o elevado coeficiente de variação no estrato E3 quando comparado com o estrato E1. Esperava-se maior volume de casca no estrato E1 em função da condição de talhadia simples, no entanto as árvores apresentaram-se menos heterogêneas, conforme se observa no coeficiente de variação reduzido (4,62\%).

O estrato E1 apresenta desvios absolutos com menores variações em relação à média, diferentemente do terceiro estrato. Fato esse devido, provavelmente, ao menor espaçamento e ao desbaste sistemático e único, realizado precocemente no primeiro estrato, contribuindo para o rápido crescimento das árvores.

A Figura 1 ilustra, graficamente, a variação do teor de casca ao longo do fuste das árvores do híbrido Eucalyptus urophylla x Eucalyptus grandis nos três estratos.

Verifica-se, na Figura 1, que há tendência de declínio na porcentagem de casca a partir da base em todos os três estratos estudados, com aumento a partir dos 9 m, à exceção do estrato E3, em que o aumento ocorreu a partir dos $6 \mathrm{~m}$. O comportamento relativo ao teor de casca deste estudo foi próximo ao encontrado por Paula Neto et al. (1992) nas espécies de Eucalyptus saligna, E. alba e E. grandis, com relação a árvores com idades entre 3 e 8 anos, em classes de DAP inferior a $30 \mathrm{~cm}$. Os resultados apontaram maior porcentagem de casca nas menores alturas, ocorrendo estabilização crescente a partir dos $4 \mathrm{~m}$ de altura. A mesma tendência de redução da porcentagem de casca nas porções intermediárias do tronco foi observada por Oliveira et al. (1996) sete espécies de eucalipto.

Os estratos apresentaram fator de forma média de 0,50; 0,52; e 0,51 nos estratos E1, E2 e E3, respectivamente. As árvores mostraram um fator de forma relativamente baixo e pouco variável entre elas, principalmente entre os estratos E1 e E3, com coeficiente de variação de $1,47 \%$ e $0,96 \%$, respectivamente, o que pode indicar que o maior espaçamento inicial disponibilizado no terceiro estrato não influenciou a forma das árvores.

Nas espécies Corymbia citriodora, Eucalyptus urophylla e E. grandis, Oliveira (1997) encontrou fatores de forma da ordem de 0,75; 0,67; e 0,62 nas árvores com 16 anos de idade e diâmetros de 24,9cm, 27,9 cm 
Tabela 3 - Valores médios do volume de casca e respectiva porcentagem das árvores em cada estrato do híbrido Eucalyptus urophylla x Eucalyptus grandis.

Table 3 - Average data for the bark volume and respective percentage for the trees in the extract for the clonal hybrid Eucalyptus urophylla $x$ Eucalyptus grandis.

\begin{tabular}{|c|c|c|c|c|c|c|}
\hline \multicolumn{6}{|c|}{ VOLUME DE CASCA $\left(\mathrm{m}^{3}\right)$} & MÉDIA \\
\hline \multicolumn{7}{|c|}{ ÁRVORES } \\
\hline 1 & 2 & 3 & 4 & 5 & & \\
\hline E1 - Talhadia(70 meses) & 0,10 & 0,09 & 0,10 & 0,10 & 0,09 & $0,10(4,62)^{*}$ \\
\hline E2 - Reforma(166 meses) & 0,12 & 0,10 & 0,10 & 0,10 & 0,11 & $0,10(7,29)$ \\
\hline E3 - Reforma(70 meses) & 0,09 & 0,08 & 0,15 & 0,09 & 0,09 & $0,10(25,65)$ \\
\hline \multicolumn{6}{|c|}{ VOLUME DE CASCA (\%) } & MÉDIA \\
\hline E1 - Talhadia(70 meses) & 10,14 & 10,25 & 10,31 & 10,62 & 9,59 & $10,18(3,68)$ \\
\hline E2 - Reforma(166 meses) & 10,57 & 9,04 & 8,62 & 8,91 & 9,70 & $9,37(8,33)$ \\
\hline E3 - Reforma(70 meses) & 10,23 & 9,51 & 15,43 & 10,93 & 10,71 & $11,36(20,59)$ \\
\hline
\end{tabular}

* Valor entre parênteses é o coeficiente de variação (\%).

* Value in parentheses is the coefficient of variation (\%).
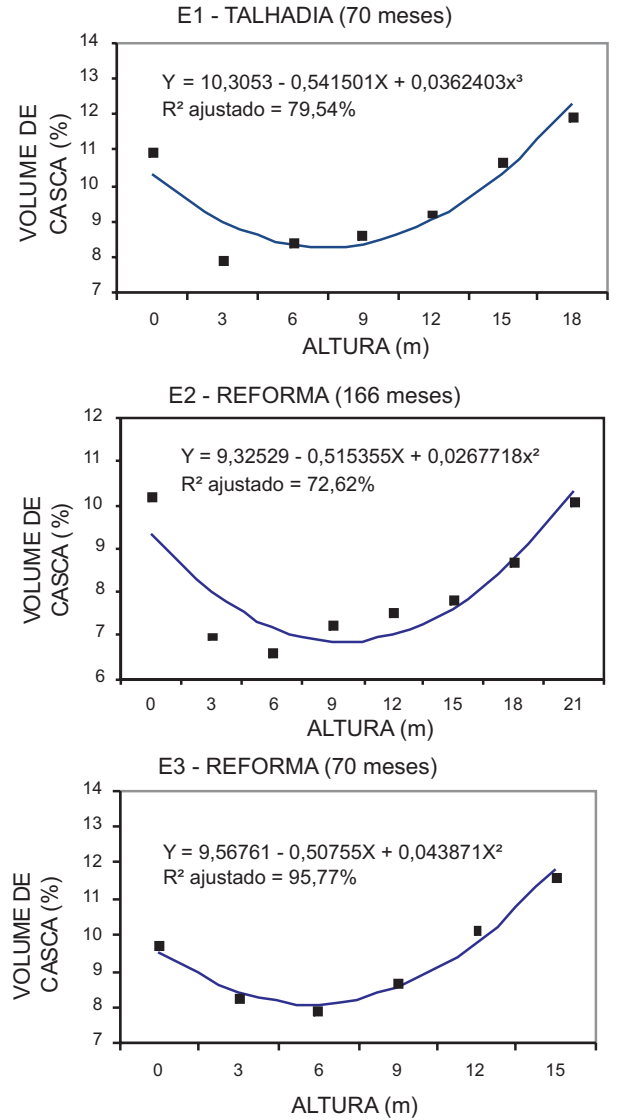

Figura 1 - Representação gráfica da variação média da porcentagem de casca ao longo do fuste nos três estratos avaliados do híbrido Eucalyptus urophylla $\mathrm{x}$ Eucalyptus grandis.

Figure 1 - Average variation of the bark percentage in the shaft of three extracts for the hybrid Eucalyptus urophylla $x$ Eucalyptus grandis. e 31,6 cm, respectivamente. Tais valores foram superiores ao encontrado neste estudo, demonstrando que o gênero Eucalyptus possui grande variabilidade de forma entre espécies, mesmo sendo o estrato E2 com maior idade.

A Tabela 4 apresenta os valores médios da conicidade em cada estrato estudado, das toras de $3 \mathrm{~m}$ de comprimento, obtidas com base nos respectivos diâmetros dos extremos das seções.

Caixeta (2000 citado por VALE et al., 2002) afirmou que, para árvores serem consideradas cônicas, a conicidade deve ser superior a $1,0 \mathrm{~cm} / \mathrm{m}$. Assim, observando a Tabela 4, à exceção da primeira tora nos três estratos, as demais se apresentaram cilíndricas.

Nas espécies Eucalyptus urophylla e E. grandis, Oliveira (1997) encontrou valores médios de conicidade de $2,02 \mathrm{~cm} / \mathrm{m}$ e $2,15 \mathrm{~cm} / \mathrm{m}$, respectivamente, na primeira tora de $4 \mathrm{~m}$, em árvores com idade aproximada de 16 anos. Neste estudo, apenas o estrato E3 aproximou-se daqueles encontrados pelo autor, sendo a baixa idade das árvores uma característica a ser destacada.

Vale et al. (2002), analisando a influência da desrama natural em Eucalyptus camaldulensis e E. urophylla em idades de 8 anos, encontraram valores médios da ordem de $0,02 \mathrm{~cm} / \mathrm{m}$ e $0,01 \mathrm{~cm} / \mathrm{m}$, respectivamente, em toras de $6 \mathrm{~m}$. Valores esses bem inferiores ao deste estudo.

Os valores de conicidade são relativamente baixos nos três estratos a partir da segunda tora (3 m). A conicidade mais elevada na primeira tora tem importância direta na produção de madeira serrada, o que poderá levar a rendimentos inferiores durante o desdobro dessas toras em relação às demais.

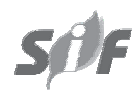

Revista Árvore, Viçosa-MG, v.34, n.5, p.947-959, 2010 
Tabela 4 - Valores médios da conicidade $(\mathrm{cm} / \mathrm{m})$ das toras de $3 \mathrm{~m}$ no fuste das árvores do híbrido Eucalyptus urophylla x Eucalyptus grandis nos três estratos.

Table 4 - Taper $(\mathrm{cm} / \mathrm{m})$ in three meter logs of the hybrid Eucalyptus urophylla $\mathrm{x}$ Eucalyptus grandis trees.

\begin{tabular}{lccc}
\hline TORA & \multicolumn{3}{c}{$\begin{array}{c}\text { CONICIDADE }(\mathrm{cm} / \mathrm{m}) \\
\text { ESTRATOS }\end{array}$} \\
\cline { 2 - 4 } & Talhadia (70 meses) & E2 & E3 \\
& $1,59(20,23) *$ & $1,76(11,18)$ & $2,02(7,89$ \\
$1[0-3 \mathrm{~m}]$ & $0,64(8,13)$ & $0,57(23,22)$ & $0,68(6,27)$ \\
$2[3-6 \mathrm{~m}]$ & $0,56(13,65)$ & $0,47(19,03)$ & $0,69(12,51)$ \\
$3[6-9 \mathrm{~m}]$ & $0,79(13,59)$ & $0,55(19,03)$ & $0,73(9,92)$ \\
$4[9-12 \mathrm{~m}]$ & $0,74(13,59)$ & $0,55(25,65)$ & $0,80(6,55)$ \\
$5[12-15 \mathrm{~m}]$ & $0,68(13,50)$ & $0,58(14,03)$ & $0,65(7,70)$ \\
$6[15-18 \mathrm{~m}]$ & $0,90(6,98)$ & $0,66(5,45)$ & - \\
$7[18-21 \mathrm{~m}]$ & - & $0,58(16,21)$ & - \\
$8[21-24 \mathrm{~m}]$ & - & & \\
\hline
\end{tabular}

* Valor entre parênteses é o coeficiente de variação (\%).

* Value in parentheses is the coefficient of variation (\%).

O índice de circularidade nas diferentes alturas do tronco pouco variaram, à exceção da posição da base, a qual apresentou os menores valores absolutos (Figura 2). A circularidade ao longo das árvores foi muito próxima entre os estratos, indicando que a idade e os diferentes sistemas de manejo aplicados não contribuíram, ou contribuíram pouco, para a formação de árvores mais circulares.

A circularidade média dos estratos E1, E2 e E3 foram, respectivamente, 0,95; 0,96; e 0,95. O modelo com melhor ajuste foi a raiz quadrada, significativo pelo teste $\mathrm{F}$ e pelo teste “t”, nos coeficientes de regressão, ambos a 5\% de probabilidade.

As árvores apresentaram circularidade média de 0,95 em todos os estratos estudados e maior heterogeneidade da base de todas as árvores. É importante ressaltar que a condição de talhadia simples do primeiro estrato não influenciou essa característica. Esperava-se que a base das árvores nessa condição apresentasse valores mais variáveis, tendo em vista a formação da madeira naquela posição, com base nas condições de manejo associadas aos estratos. Em posições superiores houve tendência de melhoria. Isso é importante quando se pretende trabalhar com madeira voltada para produtos sólidos. Árvores que apresentam circularidade muito variável podem, principalmente nas primeiras alturas, apresentar menor aproveitamento de madeira em serrarias nas fases iniciais da produção de serrados em função dos baixos rendimentos obtidos nas operações de desdobro.

Revista Árvore, Viçosa-MG, v.34, n.5, p.947-959, 2010
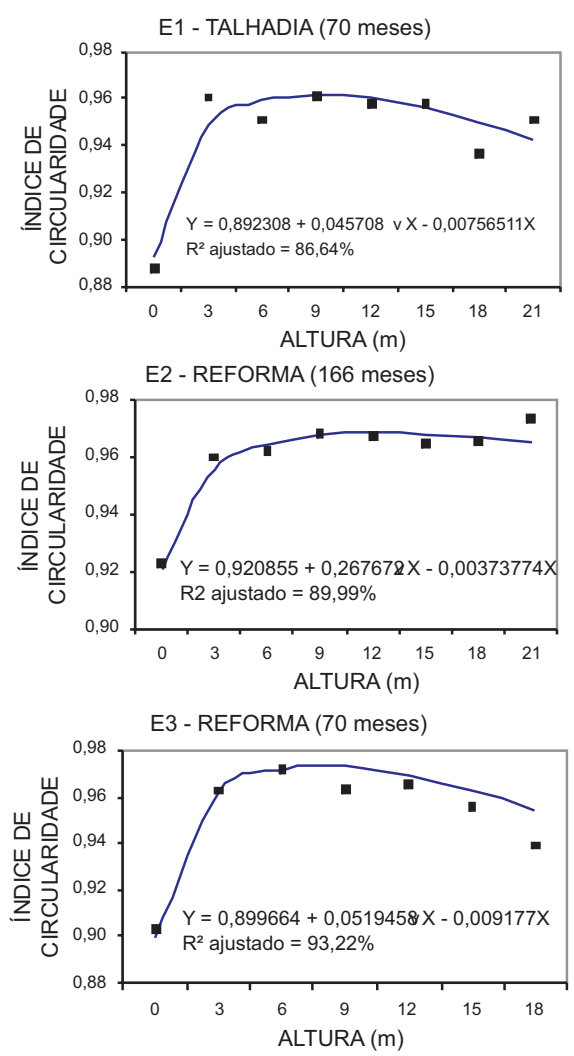

Figura 2 - Representação gráfica do Índice de Circularidade a diferentes alturas das árvores dos três estratos estudados do híbrido Eucalyptus urophylla x Eucalyptus grandis.

Figure 2 - Graphical representation of the Circularity Index of two different heights of clonal hybrid Eucalyptus urophylla $x$ Eucalyptus grandis in fifteen trees for the three extracts studied. 
Oliveira (1988), trabalhando com a espécie Xylopia sericea, espécie nativa e pioneira, encontrou um índice de circularidade médio de 0,97 em 18 árvores e mencionou que o tronco dessa espécie possui seção circular. Neste estudo, no entanto, foram encontrados valores pouco inferiores ao descrito por esse autor, podendo-se inferir que o tronco do híbrido estudado também apresenta tendência à forma circular a partir dos $3 \mathrm{~m}$. A circularidade elevada encontrada nas árvores estudadas torna-se fator positivo na sua utilização para a produção de peças serradas, uma vez que contribui para o aumento de rendimentos nas operações de desdobro.

O volume de cerne e alburno foi obtido com base nos diâmetros maior e menor em cada disco retirado nos extremos a cada seção de $3 \mathrm{~m}$ do fuste. A Tabela 5 apresenta os valores médios da espessura de alburno ao longo do tronco do híbrido Eucalyptus urophylla x Eucalyptus grandis.

Observando a Tabela 5, nota-se que o estrato E1 apresentou comportamento bem homogêneo da espessura de alburno ao longo do tronco. É interessante salientar que, mesmo apresentando proporção de alburno crescente ao longo do fuste, a espessura de alburno não variou, o que pode ser vantajoso, tendo em vista a produção de madeira onde a utilização de preservativos químicos é necessária. Os estratos E2 e E3 destacaram-se apenas na base, mantendo a espessura de alburno sem variação muito expressiva ao longo do fuste e com a proporção crescente ao longo do tronco.
Silva (2002), caracterizando a madeira de Eucalyptus grandis para a indústria moveleira, encontrou valores para a espessura de alburno inferior à deste estudo, variando de 2,5 a 3,5 cm em árvores com idades entre 10 e 25 anos, nas duas primeiras toras de $3 \mathrm{~m}$.

A Figura 5 ilustra, graficamente, o comportamento percentual de alburno e cerne ao longo do fuste das árvores estudadas. Com base na Tabela 5 e na Figura 3 (A), pode-se observar tendência de aumento da porcentagem de alburno na direção base-topo em todos os estratos. Nota-se, na Figura 3B, tendência de decréscimo da porcentagem de cerne ao longo do fuste. Comportamento similar foi observado por Oliveira (1997) em sete espécies de eucalipto. Apesar de haver outros modelos com significância a 5\% pelo teste $\mathrm{F}$ na regressão, optou-se por descrever o modelo linear por ser mais prático e representar satisfatoriamente os dados.

É fundamental salientar que, para utilização de madeira de qualidade, a quantificação do cerne e alburno é extremamente importante, uma vez que maiores proporções de cerne, por exemplo, dificultam o processo de penetração de compostos químicos, devido às suas características anatômicas, sendo preferencial para o uso em produtos serrados. Maior proporção de alburno é mais indicada quando se pretende realizar o tratamento preservativo em madeira roliça. No entanto, em indústrias à base de madeira sólida para movelaria, por exemplo, torna-se mais interessante a madeira com maiores proporções de cerne. Na Figura 3, pode-se observar que em todos os estratos a proporção de cerne manteve a mesma tendência em se reduzir com o aumento da altura.

Tabela 5 - Valores médios da espessura de alburno (cm) e respectiva proporção (\%) ao longo do tronco do híbrido Eucalyptus urophylla x Eucalyptus grandis nos estratos estudados.

Table 5 - Average sapwood thickness (cm) and respective proportion (\%) along the clonal hybrid Eucalyptus urophylla $x$ Eucalyptus grandis trunk in the studied extracts.

\begin{tabular}{|c|c|c|c|c|c|c|}
\hline \multirow{2}{*}{$\begin{array}{l}\text { ALTURADO } \\
\text { TRONCO (m) }\end{array}$} & \multicolumn{2}{|c|}{ E1Talhadia(70 meses) } & \multicolumn{2}{|c|}{ E2Reforma(166 meses) } & \multicolumn{2}{|c|}{ E3Reforma(70 meses) } \\
\hline & $(\mathrm{cm})$ & $(\%)$ & $(\mathrm{cm})$ & $(\%)$ & $(\mathrm{cm})$ & $(\%)$ \\
\hline Base0 & $2,85(9,55)^{*}$ & $36,22(3,38)$ & $3,39(21,36)$ & $28,03(9,31)$ & $3,40(19,85)$ & $37,03(4,76)$ \\
\hline 3 & $2,87(4,76)$ & $41,73(5,98)$ & $1,98(10,63)$ & $28,60(8,78)$ & $2,51(7,34)$ & $37,55(4,90)$ \\
\hline 6 & $3,06(12,93)$ & $44,77(6,92)$ & $1,89(11,36)$ & $29,21(7,80)$ & $2,54(10,31)$ & $40,48(3,41)$ \\
\hline 9 & $2,88(9,08)$ & $47,06(3,67)$ & $1,82(8,63)$ & $29,84(7,75)$ & $2,49(3,53)$ & $43,94(2,92)$ \\
\hline 12 & $2,83(5,49)$ & $51,22(12,60)$ & $1,72(8,92)$ & $31,31(5,38)$ & $2,50(6,62)$ & $48,96(4,64)$ \\
\hline 15 & $2,77(6,07)$ & $56,56(6,14)$ & $1,81(4,43)$ & $35,21(6,39)$ & $2,56(7,65)$ & $55,27(6,25)$ \\
\hline 18 & $2,87(10,64)$ & $66,25(3,69)$ & $1,84(13,30)$ & $38,32(8,66)$ & $2,65(5,45)$ & $59,72(-)$ \\
\hline 21 & - & - & $1,76(5,08)$ & $41,33(8,29)$ & - & - \\
\hline MÉDIAS & $2,91(9,29)$ & & $1,99(28,93)$ & & $2,65(15,59)$ & \\
\hline
\end{tabular}

* Valor entre parênteses é o coeficiente de variação (\%).

* Value in parentheses is the coefficient of variation (\%). 

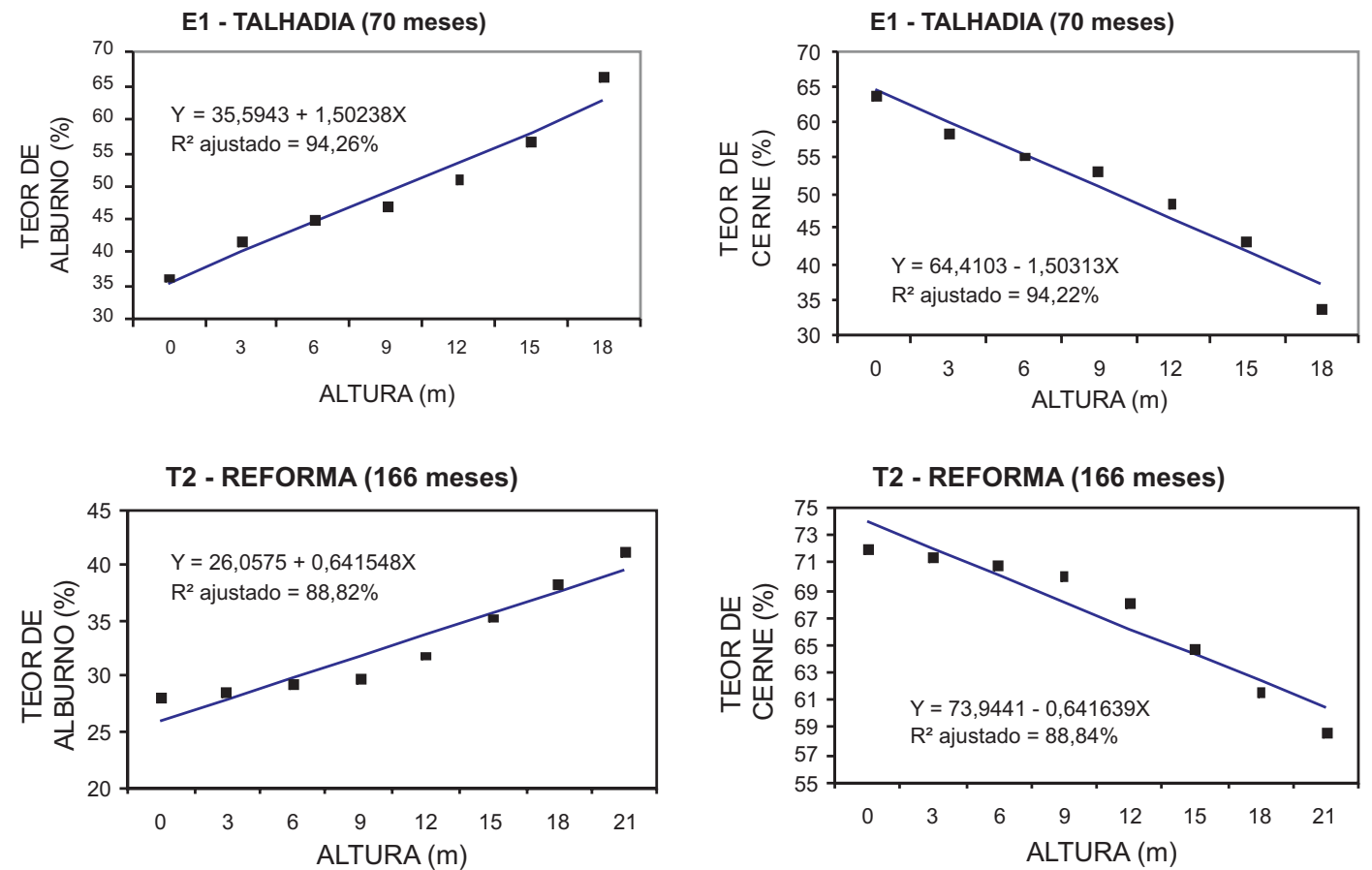

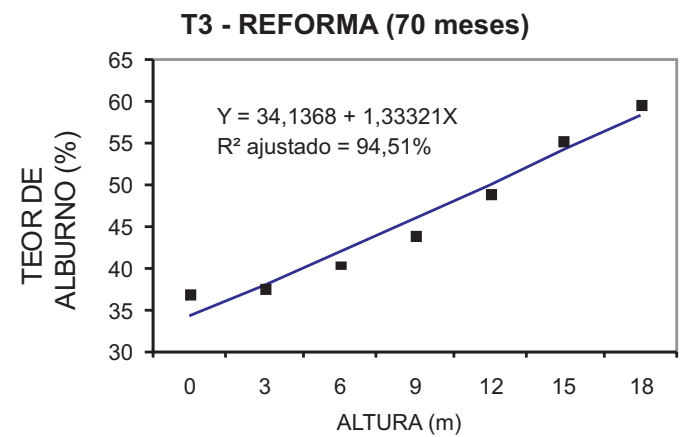

(A)

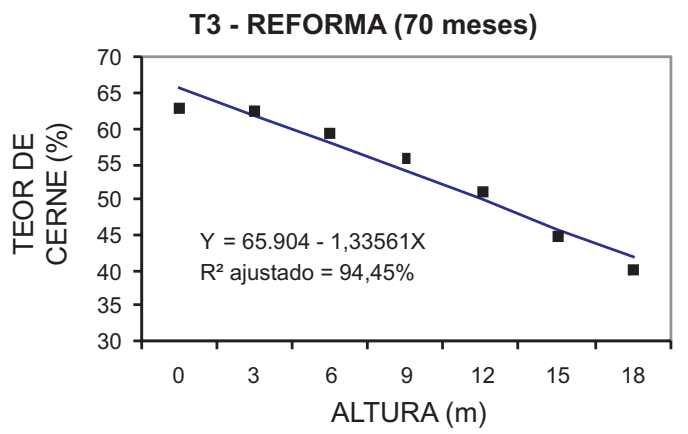

(B)

Figura 3 - Representação gráfica da porcentagem média de alburno (A) e cerne (B) dos três estratos avaliados ao longo do tronco do híbrido Eucalyptus urophylla x Eucalyptus grandis com as respectivas equações ajustadas.

Figure 3-Graphical representation of the medium percentage of sapwood (A) and heartwood (B) in the three appraised extracts along the trunk of the clonal hybrid Eucalyptus urophylla $x$ Eucalyptus grandis and respective adjusted equations.

O primeiro estrato apresentou os melhores resultados absolutos para a relação cerne-alburno, destacando-se a primeira tora, em comparação com o terceiro estrato. No estrato E2, apesar da maior idade, o crescimento foi mais lento, contribuição essa devida, provavelmente, aos dois desbastes ocorridos tardiamente, fornecendo condições para maior proporção de cerne em relação ao alburno.
O maior espaçamento $(6,0$ x 2,5 m) permitiu a formação de maior porcentagem de alburno na primeira tora em razão, provavelmente, do desbaste realizado na época certa (3,5 anos) com as árvores obtendo crescimento satisfatório em diâmetro. No entanto, a relação cernealburno apresentou-se pouco superior no primeiro estrato com a mesma idade e desbaste realizado precocemente (2,5 anos). Salienta-se que, em função do sistema de

Revista Árvore, Viçosa-MG, v.34, n.5, p.947-959, 2010 
talhadia simples, o sistema radicular já estava formado, o que pode ter contribuído para o rápido crescimento das árvores e a formação do lenho.

Em ciclos de corte mais prolongados há incremento em diâmetro das toras e, consequentemente, mais rendimento em madeira de qualidade superior, haja vista a maior porcentagem de cerne (SELLA, 2001 citado por SILVA, 2002). Este estudo comprovou que, na idade de 166 meses (E2 - condição de reforma), houve maior proporção de cerne, e a primeira tora apresentou valores bem próximos ao da segunda tora, à exceção do primeiro estrato cultivado na condição de talhadia simples, em que os valores foram superiores.

Na Tabela 6, encontram-se os resultados dos coeficientes de Pearson, provenientes da correlação linear entre algumas propriedades físicas e mecânicas e das características dendrométricas avaliadas do híbrido clonal de Eucalyptus urophylla x Eucalyptus grandis.

A densidade básica apresentou forte correlação negativa com o fator anisotrópico $(-0,83)$, a espessura de alburno $(-0,84)$ e a conicidade $(-0,75)$. Por consequência, houve forte correlação positiva com as contrações nas direções tangencial $(0,80)$ e radial $(0,92)$, bem assim com a volumétrica $(0,93)$.

Adorno e Garcia (2003) não encontraram correlação significativa entre a densidade básica e a conicidade em árvores de Eucalyptus grandis e E. urophylla, ambos com 8 anos de idade e plantados no espaçamento de 2,0 x 3,0 m. Neste trabalho, em função da boa correlação da conicidade com as demais variáveis estudadas, pode-se inferir que ela apresenta potencial satisfatório no processo de seleção genética.

Clair et al. (2003) não encontraram correlação significativa entre a densidade básica e a DRL na espécie Castanea sativa, observando correlação inferior $(0,11)$ à deste estudo (0,32). Lima et al. (2004), estudando a DRL associada a outras propriedades da madeira de clones de Eucalyptus urophylla x Eucalyptus grandis, afirmaram que não houve correlação significativa entre a DRL e a densidade básica, a contração volumétrica, a compressão paralela às fibras, o MOE e o MOR em nenhum dos clones avaliados.

Oliveira (1997), estudando a correlação entre a retratibilidade volumétrica total e a densidade básica em sete espécies de eucalipto com 16 anos de idade, encontrou correlação significativa a $1 \%$ de probabilidade

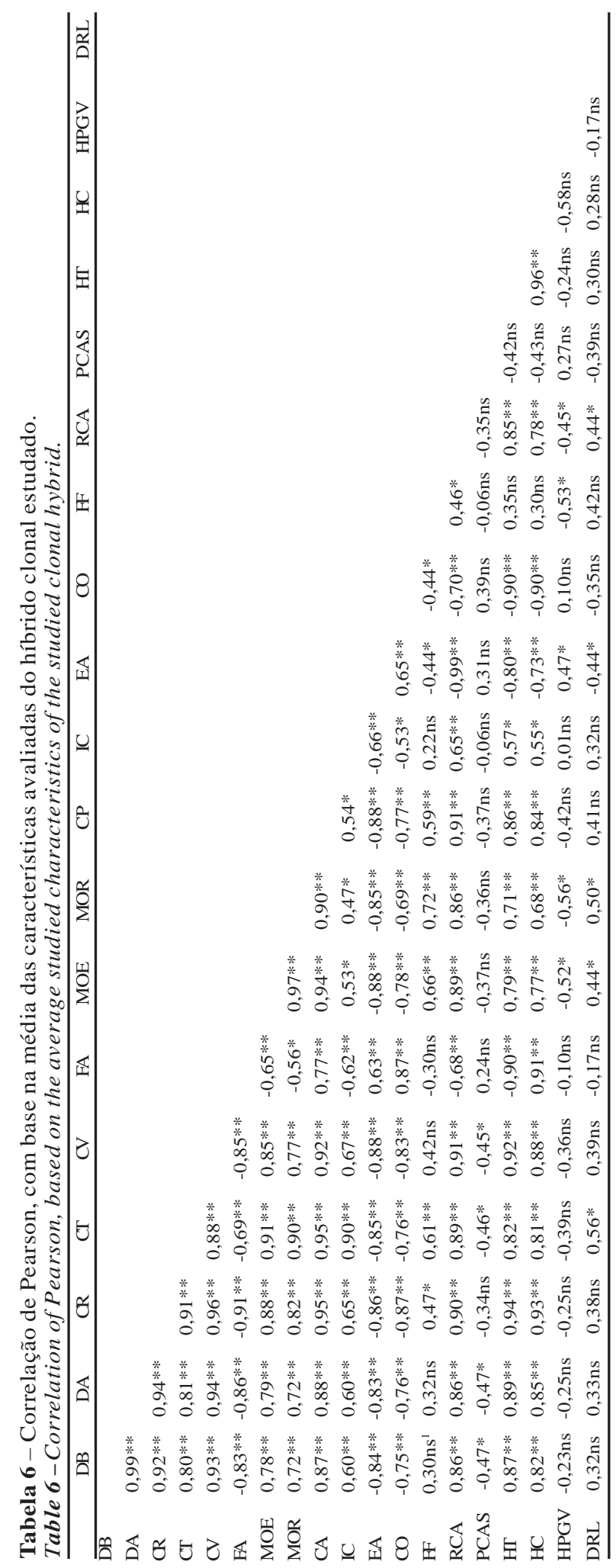

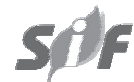

Revista Árvore, Viçosa-MG, v.34, n.5, p.947-959, 2010 
entre a contração volumétrica e a densidade básica $(0,77)$, na espécie Eucalyptus grandis, não verificando correlação na espécie E. urophylla.

Panshin e De Zeeuw (1964) relataram a existência de forte correlação entre a densidade e as propriedades de resistência da madeira. Neste estudo, as propriedades mecânicas (MOR e MOE) apresentaram fortes correlações positivas com todas as propriedades físicas avaliadas, sendo negativas e significativas com o fator anisotrópico (-0,65 e -0,56, respectivamente). Adorno e Garcia (2003) não constataram correlação significativa na compressão paralela às fibras com o módulo de elasticidade na espécie Eucalyptus grandis. No entanto, na espécie E. urophylla esses autores encontraram correlação positiva de 0,35, significativa a apenas $0,01 \%$. Neste, a compressão paralela às fibras apresentou, como esperado, forte correlação significativa com o $\operatorname{MOE}(0,77)$.

Lima (1996 citado por OLIVEIRA, 1997), estudando as correlações entre contrações e densidade para a madeira de Eucalyptus pilularis e E. microcorys de 21 anos de idade, encontrou um coeficiente de correlação de $-0,36$ e 0,84 , respectivamente, entre a contração volumétrica total e a densidade básica, em um nível de probabilidade de 5\%. Para a madeira do híbrido clonal de Eucalyptus urophylla x Eucalyptus grandis deste estudo, essa mesma correlação foi significativa no nível de significância de 1\%, apresentando coeficiente de correlação igual a 0,93.

Cruz et al. (2003), avaliando a variação das propriedades físicas e mecânicas da madeira de sete clones de Eucalyptus com idades de 5,5 e 10,5 anos em diferentes espaçamentos, encontraram fortes correlações entre o MOR, o MOE e a densidade básica, com destaque para a segunda tora. Foram avaliados clones das espécies Eucalyptus urophylla e E. camaldulensis, em que se destacou um clone da espécie E. urophylla, por apresentar o menor coeficiente de anisotropia (razão entre as contrações tangenciais e radiais), cultivado no espaçamento de 10,0 x 4,0 m, com diâmetro de 26,5 cm e idade de 5,5 anos.

A Tabela 7 apresenta o coeficiente de Pearson para algumas propriedades físicas e variáveis dendrométricas estudadas em função das alturas em seções de $3 \mathrm{~m}$, para o híbrido clonal de Eucalyptus urophylla x Eucalyptus grandis.

Na Tabela 7, pode-se observar que a densidade tende a um aumento no sentido longitudinal das árvores, seguida de incremento negativo para o fator anisotrópico e conicidade. Não foram encontradas correlações significativas para a altura em função das contrações lineares e volumétricas, assim como para a circularidade, espessura de alburno e relação cerne-alburno.

A Tabela 8 mostra o coeficiente de Pearson para algumas propriedades físicas e mecânicas em função da posição radial nas toras do híbrido clonal estudado.

A densidade básica apresentou-se superior na primeira tora, com tendência de aumento linear no sentido medulacasca, sendo altamente significativa (Tabela 8). Nota-se ainda que a contração radial apresenta melhor correlação em relação à tangencial para a primeira tora no sentido medula-casca, positiva e significativa e com tendência de aumento. A segunda tora não apresentou o mesmo comportamento. Com relação à contração volumétrica, existe tendência em haver aumento na direção medulacasca para as toras, destacando-se a tora 1. Oliveira (1997), estudando as correlações entre a retratibilidade volumétrica na posição radial em sete espécies de eucalipto, encontrou correlações significativas e positivas com tendência de aumento na direção radial das espécies Eucalyptus cloeziana (0,75), E. urophylla $(0,28)$, E. grandis $(0,65)$ e Corymbia citriodora $(0,39)$, valores superiores ao encontrado por este estudo, à exceção da primeira tora e da espécie E. urophylla.

Tabela 7 - Coeficientes de Correlação de Pearson $(\mathrm{p}<0,01)$ entre a altura total e algumas propriedades físicas e dendrométricas das árvores do híbrido clonal de Eucalyptus urophylla x Eucalyptus grandis.

Table 7 - Correlation of Pearson $(p<0.01)$ between height and some physical properties and dendrometrics in trees of the clonal hybrid of Eucalyptus urophylla x Eucalyptus grandis.

\begin{tabular}{cccccccccc}
\hline DB & DAN & CR & CT & CV & FA & IC & EA & CO & RCA \\
\hline 0,63 & 0,49 & $n^{1}$ & ns & ns & $-0,85$ & ns & ns & $-0,51$ & ns \\
\hline
\end{tabular}

${ }^{1}$ Não significativo.

${ }^{1}$ Not significant. 
Tabela 8 - Coeficientes de Correlação de Pearson $(\mathrm{p}<0,01)$ entre algumas propriedades físicas e mecânicas e a posição radial do tronco das duas primeiras toras de $3 \mathrm{~m}$ das árvores do híbrido clonal de Eucalyptus urophylla x Eucalyptus grandis.

Table 8 - Correlation of Pearson $(p<0.01)$ among some physical and mechanical properties and the radial position of the trunk for the first two logs of three meters in trees of the clonal hybrid of Eucalyptus urophylla x Eucalyptus grandis.

\begin{tabular}{|c|c|c|c|c|c|c|c|c|}
\hline \multirow{2}{*}{$\overline{\text { TORA }}$} & \multicolumn{5}{|c|}{ Propriedades Físicas } & \multicolumn{3}{|c|}{ Propriedades Mecânicas } \\
\hline & DB & CR & CT & CV & FA & MOR & MOE & CP \\
\hline 1 & 0,46 & 0,30 & 0,22 & 0,32 & 0,16 & 0,47 & 0,45 & $\overline{0,44}$ \\
\hline 2 & 0,39 & 0,27 & $\mathrm{~ns}^{1}$ & 0,20 & ns & 0,45 & 0,37 & 0,23 \\
\hline
\end{tabular}

${ }^{1}$ Não significativo.

${ }^{1}$ Not significant.

O comportamento do coeficiente de anisotropia da madeira do híbrido clonal deste estudo apresentou tendência de aumento na direção medula-casca. Apesar de altamente significativo, Oliveira (1997) chamou atenção para o fato de o gênero Eucalyptus apresentar grande número de espécies e o comportamento da madeira ser muito variável entre elas e mesmo dentro da mesma árvore. Esse autor encontrou correlações significativas e negativas a $1 \%$ de probabilidade para cinco de sete espécies de eucalipto estudadas, contrastando com esta pesquisa.

As propriedades mecânicas avaliadas apresentaram tendência de aumento na direção medula-casca, com melhores coeficientes de correlação na primeira tora, indicando que as melhores propriedades de resistência à flexão estática e à resistência à compressão estejam nas posições mais afastadas da medula, consequentemente na região de madeira mais adulta. Deve-se atentar para o fato de que, durante o desdobro das peças, o descarte das costaneiras deve ser minimizado, isto é, mais bem aproveitado.

Em razão de as contrações radiais se apresentarem maior e com tendência de aumento na direção medulacasca, sugere-se que o desdobro, principalmente da primeira tora, seja realizado de forma radial, o que poderia minimizar possíveis defeitos, como rachaduras e empenamentos, tendo em vista o fato de o processo de secagem ser realizado mais facilmente.

\section{CONCLUSÕES}

Com base nos resultados, nas considerações expostas e nas condições em que foi realizado o experimento, puderam-se obter as seguintes conclusões:

- Os diferentes sistemas de manejo aplicados aos três estratos permitiram que o desenvolvimento em diâmetro das árvores fosse similar, contribuindo para a formação de cerne e alburno muito próximo entre os estratos.
- O estrato E1 destacou-se com a maior média absoluta em espessura de alburno. Em contrapartida, o estrato E2 apresentou os maiores valores médios em volume de cerne das árvores proporcionado provavelmente pelos dois desbastes tardios e pela maior idade.

- Entre as árvores de mesma idade, cultivadas em sistemas de plantio e manejo diferenciados (E1 e E3), destacaram-se as primeiras toras para a proporção cerne-alburno, sendo encontrados no primeiro estrato os maiores valores absolutos.

- As árvores de todos os estratos apresentam forma tendendo à cônica ao longo do fuste.

- A tora um de todos os estratos estudados apresentou-se cônica, e as demais exibiram comportamento cilíndrico, indicando um bom rendimento para a serraria a partir da segunda tora.

- As propriedades de resistência mecânica (MOE e MOR) e resistência à compressão paralela às fibras apresentaram forte correlação com a densidade básica e as contrações lineares e volumétricas.

- Entre as duas primeiras toras avaliadas, a segunda tora não apresentou correlação significativa para o fator anisotrópico na direção radial, indicando melhor estabilidade dimensional, que mediante o processo de desdobro e secagem corretos poderá proporcionar peças de melhor qualidade.

- Não foram encontradas correlações significativas entre as contrações radial e tangencial e volumétrica longitudinalmente na árvore, mas sim, radialmente, mostrando a importância de se conhecerem as variações dessas propriedades no sentido medula-casca da madeira, a fim de formar lotes mais homogêneos.

- Esperavam-se melhores correlações, principalmente entre a conicidade e o fator de forma, por estarem diretamente ligados à liberação de tensões durante o desdobro. 


\section{REFERÊNCIAS}

ADORNO, M. F. C.; GARCIA, J. N. Correlações lineares entre as principais características tecnológicas da madeira de clones de Eucalyptus grandis e Eucalyptus urophylla. Scientia Forestalis, n.63, p.44-53, 2003.

\section{ASSOCIAÇÃO BRASILEIRA DE NORMAS} TÉCNICAS - ABNT. Ensaios físicos e mecânicos da madeira. MB-26/40. Rio de Janeiro: 1940. 16p.

BERGER, R. B. et al. Efeito do espaçamento e da adubação no crescimento de um clone de Eucalyptus saligna Smith. Ciência Florestal, v.12, n.2, p.75-87, 2002.

CLAIR, B.; RUELLE, J.; THIBAUT, B. Relationship between growtrh stresses, mechano-physical properties and proportion of fibre with gelatinous layer in Chestnut (Castanea sativa Mill.). Holzforchung, v.57, p.189-195, 2003.

CRUZ, C. R.; LIMA, J. T.; MUNIZ, G. I. B. Variações dentro das árvores e entre clones das propriedades físicas e mecânicas da madeira de híbridos de Eucalyptus. Scientia Forestalis, v.64, p.33-37, 2003.

FINGER, C. A. G. et al. Efeito da intensidade de desrama sobre o crescimento e a produção de Eucalyptus saligna Smith. Cerne, v.7, n.2, p.53-64, 2001.

GONÇALVES, J. L. M. et al. Silvicultural effects on the productivity and wood quality of eucalypt plantations. Forest Ecology Management, v.193, p.45-61, 2004.

LEITE, H. G.; NOGUEIRA, G. S.; DIAS, A. N. Desbastes e seus efeitos sobre a produção e qualidade de povoamentos florestais. In: SEMINÁRIO SOBRE SILVICULTURAEM FLORESTAS PLANTADAS, 1., 2004, Vitória. Anais... Viçosa: Sociedade de Investigações Florestais/SIF, 2004. p.165-195.

LIMA, J. T. et al. Deformações residuais decorrentes de tensões de crescimento em eucaliptos e suas associações com outras propriedades. Revista Árvore, v.28, n.1, p.107-116, 2004.
OLIVEIRA, J. T. S. Caracterização da madeira de eucalipto para a construção civil. 1997. 429f. Tese (Doutorado em Engenharia Civil) - Escola Politécnica da Universidade de São Paulo, São Paulo, 1997.

OLIVEIRA, J. T. S. Estudo das propriedades físicas e tecnológicas da madeira da Pindaíba (Xylopia sericea St. Hill.). 1988. 106f. Dissertação (Mestrado em Ciências Florestais) - Universidade Federal de Viçosa, Viçosa, MG,1988.

OLIVEIRA, J. T. S. et al. Caracterização da madeira de sete espécies de eucaliptos para a construção civil: 1- Avaliações dendrométricas das árvores. Scientia Forestalis, n.56, p.113-124, 1996.

PANSHIN, A. J.; DE ZEEUW, C. Textbook of wood tecnology. 2.ed. New York: McGraw Hill, 1964. 643p. v.1

PAULA NETO, F. et al. Variação da percentagem de casca ao longo do tronco e entre árvores de eucalipto. Revista Árvore, v.16, n.3, p.309318, 1992.

PINHEIRO, A. L. Considerações sobre taxonomia, filogenia, ecologia, genética, melhoramento florestal e a fertilização mineral e seus reflexos na anatomia da madeira. Viçosa, MG: SIF, 1999. 144p.

SCANAVACA JR., L.; GARCIA, J. N. Rendimento em madeira serrada de Eucalyptus urophylla. Scientia Forestalis, n.63, p.32-43, 2003.

SCHÖNAU, A. P. G.; COETZEE, J. Initial spacing, stand density and thinning in eucalypt plantations. Forest Ecology Management, v.29, p.245-266, 1989.

SCOLFORO, J. R.; FIGUEIREDO FILHO, A.

Mensuração florestal. Módulo 2: volumetria. Lavras: ESAL/FAEPE, 1993.126p.

SILVA, J. C. Caracterização da madeira de Eucalyptus grandis Hill ex. Maiden, de diferentes idades, visando a sua utilização na indústria moveleira. 2002. 160f. Tese (Doutorado em Ciências Florestais) - Universidade Federal do Paraná, Curitiba, 2002. 
VALE, R. S. et al. Efeito da desrama artificial na qualidade da madeira de clones de eucalipto em sistema agrossilvipastoril. Revista Árvore, v.26, n.3, p.285-297, 2002.

VITAL, B. R. Métodos de determinação da densidade da madeira. Viçosa, MG: SIF, 1984. 21 p. (Boletim Técnico, 1).
WILKINS A. P.; HORNE, R. Wood-density variation of young plantation-grown Eucalyptus grandis in response to silvicultural treatments. Forest Ecology Management, v.40, p.39-50, 1991.

ZOBEL, B. Silvicultural effects on wood properties. IPEF International, n.2, p.31-38, 1992. 